\title{
EXCHANGE COUPLED DOUBLE LAYERS FOR MAGNETO-OPTICAL APPLICATIONS
}

\author{
S. Becker, H. Rohrmann, K. Roell \\ FB Physik, Universitaet Kassel, 34109 Kassel, Germany \\ AND F. STOBIECKI \\ Institute of Molecular Physics, Polish Academy of Sciences \\ Smoluchowskiego 17/19, 60-179 Poznan, Poland
}

Optical data storage is a rapidly developing technique, which emerges in many varieties. Magneto-optical recording, in particular, applies amorphous rare earth/transition metal films, where a binary coded information is written thermomagnetically and is read by the magneto-optical Kerr or Faraday effect. To increase the writing speed and the recording density, techniques for direct overwrite and magnetically induced superresolution were proposed, which involve these of exchange coupled double layer systems as a storage media. In this paper, the principles and the properties of exchange coupled double layers are discussed with respect to the direct overwrite and the magnetically induced superresolution processes. A formalism based on a switching field diagram is developed yielding all information necessary to achieve reliable and practical writing procedures. As an example, the direct overwrite capability is demonstrated experimentally in antiparallel coupled layer systems.

PACS numbers: $42.79 . \mathrm{Vb}, 78.20 . \mathrm{Ls}, 75.70 .-\mathrm{i}, 75.70 . \mathrm{Ch}$

\section{Introduction}

Optical recording is an emerging technique which competes with the widely used magnetic recording in many areas of data storage. In a consumer product it has started with the compact audio disc (CD) and is now developing in many varieties, for instance CD-ROMs, write-once-read-many media (WORMs) or magneto-optical minidisk. The various optical recording techniques differ mainly in the way how the information is written and detected. In magneto-optical recording, in particular, the data are written and erased thermomagnetically and are read by the magneto-optical Kerr or Faraday effect [1-4]. The reversible erasing process allows an almost unlimited number of writing cycles.

In the thermomagnetic writing process a homogeneously magnetized medium, which may represent the information " 1 " is heated locally by a short laser pulse to a temperature near the Curie temperature (Fig. 1). If an oppositely oriented bias 


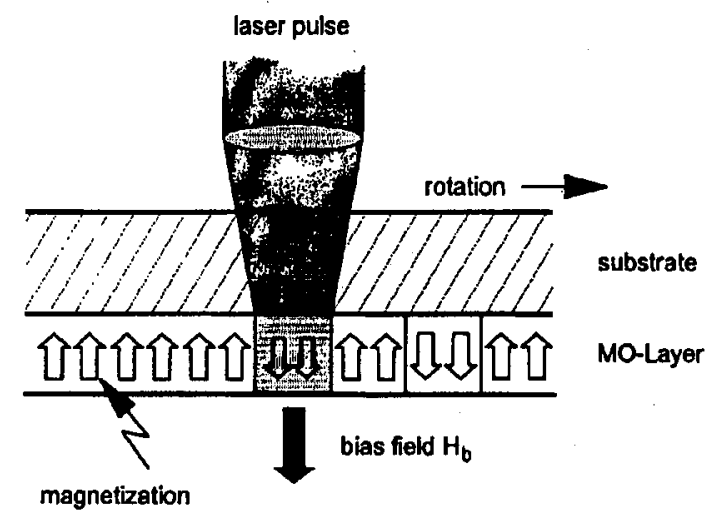

Fig. 1. Principle of magneto-optical recording.

field $H_{\mathrm{b}}$ is present during cooling, a magnetic domain is formed, which may represent the information " 0 ". This process requires a medium with a perpendicular anisotropy and a sufficiently small Curie temperature (about $220^{\circ} \mathrm{C}$ ). It is achieved by amorphous thin films consisting of rare earth metals (RE) and transition metals (TM) which have an antiferromagnetic coupling. Examples are $\mathrm{TbFe}, \mathrm{TbFeCo}$ or GdTbFe. The magnetic properties of these films can be adjusted in a wide range by the composition $[4,5]$. The size of the domain is determined by the temperature profile $T(r)$ and by the profile of the temperature dependent coercivity $H_{\mathrm{c}}(r)$. The stability of the domain is influenced by the wall energy, the magnetization and the anisotropy energy. It should be noted that the size of the written domain can be smaller than the diffraction limit of the laser beam (see Sec. 6).

The reading process is performed by the magneto-optical Kerr effect, which transforms the linear polarization of a low power reading laser into an elliptical polarization tilted by a small Kerr angle $\Theta_{\mathrm{K}}$, when the light is reflected from a magnetized medium. The Kerr rotation and ellipticity are detected by a polarizer which transforms the change of polarization into a modulation of light intensity.

For writing new information firstly a high intensity laser is applied to the whole track in presence of a bias field, to magnetize the film homogeneously along to track. This erasing procedure (initialization) requires a full rotation of the disk and limits the data access time. Research is concentrated, therefore, to circumvent this procedure by appropriate layer systems which allow direct overwrite (DOW). A solution proposed firstly by Saito et al. [6] applies exchange coupled double layers (ECDLs) as storage media and a laser modulation technique with three power levels: a very low reading power and two different laser powers for writing at high temperature $T_{\mathrm{H}}$ or low temperature $T_{\mathrm{L}}$.

\section{Exchange coupled double layer (ECDL)}

As an example, Fig. 2 shows magnetization configurations of an ECDL having antiparallel coupling [7]. It consists of a layer (1), which is dominated by the subnetwork magnetization of the TM component and a layer (2), which is dominated by the RE component. Due to exchange coupling, the subnetwork 
a)

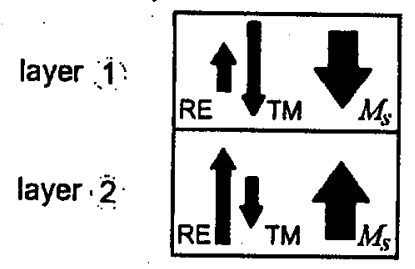

b)

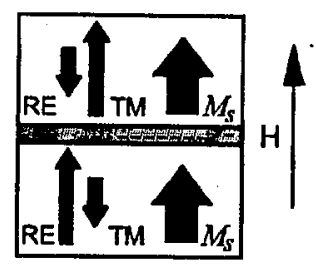

Fig. 2. Subnetwork magnetizations of the RE- and TM components and net magnetization $M_{s}$ of exchange coupled double layers with antiparallel coupling; (a) magnetic configuration in the ground state without external field; (b) magnetic configuration in a saturation field.

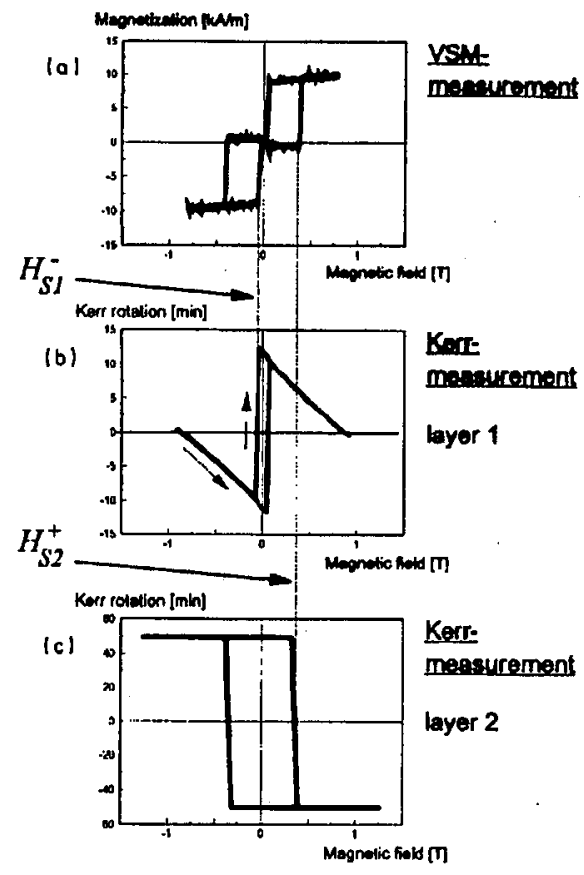

Fig. 3. Hysteresis loops of an exchange coupled double layer $\mathrm{TbFe} / \mathrm{TbFeCo}$; (a) VSM measurement; $(b, c)$ Kerr measurement from the substrate and surface side, respectively.

magnetizations of RE and TM are continuous, but the net magnetizations $M_{\mathrm{s}}$ of layer (1) and layer (2) are opposite to each other (antiparallel coupling). In a saturation field, however, both net magnetizations will be turned into the direction of the field. As a consequence, the subnetwork magnetizations form an interface wall, which contains the wall energy $\sigma_{\mathrm{w}}$ per unit area. If the coercivity of the layers is sufficiently high, this is a stable configuration. A switching field may be required to return to the original state. In this case, the wall is annihilated and the wall energy $\sigma_{\mathrm{w}}$ is released. Therefore, the switching field for a magnetic 
reversal is given by [7-9]:

$$
H_{\mathrm{s}}^{ \pm}=3 D H_{\mathrm{c}} \pm \sigma_{\mathrm{w}} / 2 M_{\mathrm{s}} t \text {. }
$$

The first term describes the coercivity $H_{\mathrm{c}}$ required to reverse an uncoupled single layer having thickness $t$ and magnetization $M_{\mathrm{s}}$; the second term represents the coupling by the wall energy. The sign $(t)$ is related to creation of a wall and the sign (-) to wall annihilation.

The switching fields can be measured by means of a vibrating sample magnetometer VSM (Fig. 3a) or by Kerr measurements (Fig. 3b and c). In case of Kerr magnetometry, the hysteresis loops of both films in the stack can be determined separately by measurements either from the surface side or through the glass substrate. The VSM measurements provide a superposition of the hysteresis loops of both films. The slope in Fig. 3b is caused by the Faraday effect of the glass. The inverted sign of the hysteresis loop in Fig. $3 \mathrm{c}$ is due to the fact that the film is RE dominated, but the Kerr effect is sensitive only to the TM component.

Similar measurements can be performed at various temperatures. In this way the switching fields $H_{\mathrm{s}}^{ \pm}$can be determined as a function of temperature for each individual film in the stack (see Sec. 4). Note that in the particular case of Fig. 3b the switching takes place already before the moment, when the magnetic field has changed its sign. The energy for switching against the applied external field comes from the release of wall energy. This situation, which corresponds to a negative switching field, is an important perquisite for the direct overwrite (see Sec. 4).

\section{Principle of direct overwrite}

Figure 4 shows how ECDLs with antiparallel coupling can be applied for a direct overwrite process [6]. The medium consists of a memory layer (1) dominated by the TM component and a reference layer (2) dominated by the RE component. Since the coupling is antiparallel, the net magnetizations of layer (1) and layer (2) are opposite to each other. The information is read only from the memory layer, whereas the reference layer is used to control the writing process. The principle idea is that an initialization performed at room temperature should be followed by a writing process at an elevated temperature $T_{\mathrm{L}}$ or $T_{\mathrm{H}}$. During operation, therefore, the recording disk runs firstly through a region, where a permanent initialization field $H_{\text {ini }}$ is applied and then through a region where the writing takes place by low or high power laser pulses in presence of an opposite permanent bias field $H_{\mathrm{b}}$.

The initialization field $H_{\text {ini }}$ turns the magnetization of the reference layer (2) homogeneously into field direction but must not change the magnetization of the memory layer (1). This requires a low coercivity $H_{\mathrm{c} 2}$ of the reference layer and a high coercivity $H_{\mathrm{c} 1}$ of the memory layer at room temperature.

A second condition refers to the Curie temperature of the reference layer (2), which should be higher than that of the memory layer (1). A low power laser pulse may heat the memory layer (1) to a temperature $T_{\mathrm{L}}$ close to its Curie temperature, but does not significantly influence the reference layer. During cooling a domain is formed in the memory layer, which is opposite to that of the reference layer due to the exchange energy. As a consequence, the information " 0 " is written into the memory layer independent of the former information. 


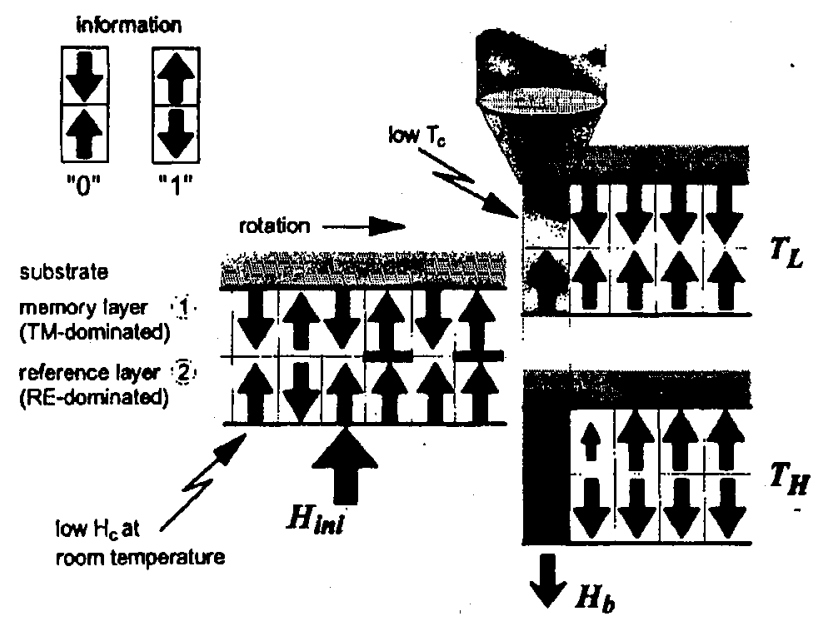

Fig. 4. Principle of direct overwrite in an ECDL with antiparallel coupling; the film is initialized by a field $H_{\text {ini }}$ at room temperature and is then exposed to a low or high power laser pulse in presence of a bias field $H_{\mathrm{b}}$; the cycle at low temperature $T_{\mathrm{L}}$ leads to a configuration representing " 0 " whereas the cycle at high temperature $T_{\mathrm{H}}$ leads to " 1 ".

Alternatively, a high power laser pulse is applied to heat the layer system up to a temperature $T_{\mathrm{H}}$ close to the Curie temperature of the reference layer (2). Then the bias field $H_{\mathrm{b}}$ switches firstly the magnetization of layer (2). During cooling a domain is formed also in the memory layer (1) the magnetization of which is opposite to that of the reference layer (and opposite to the bias field) due to the exchange coupling. Eventually, a magnetization configuration results, which represents the information " 1 ".

The conditions required for these processes, i.e. $H_{\mathrm{c} 2}<H_{\mathrm{c} 1}$ at room temperature and $T_{\mathrm{c} 1}<T_{\mathrm{c} 2}$, can be adjusted by appropriate film compositions [5]. Typical values are $\mathrm{Tb}_{22} \mathrm{Fe}_{78}$ for the TM dominated memory layer (1) and $\mathrm{Tb}_{30}\left(\mathrm{Fe}_{90} \mathrm{Co}_{10}\right)_{70}$ for the $\mathrm{RE}$ dominated reference layer (2). Then, a temperature dependence of the $H_{\mathrm{c}}$ as shown in Fig. 5 results for the uncoupled single layers.

\section{Magnetic reversal processes in ECDLs}

Many authors try to determine the switching conditions for DOW only from the coercivities $H_{\mathrm{c}}(T)$ as shown in Fig. 5. During a DOW cycle, however, interface walls are created and annihilated the energy of which has to be taken into account for a more detailed discussion. The diagram, therefore, is modified significantly, when exchange coupled double layers are formed by a combination of single layers [9]. An example is shown in Fig. 6.

Below a critical temperature $T_{\mathrm{s}} \approx 55^{\circ} \mathrm{C}$ firstly the reference layer (2) is switched and then the memory layer (1). Above the critical temperature the sequence is opposite: the memory layer switches firstly and the reference layer switches secondly. Note that in a certain temperature range from $70^{\circ} \mathrm{C}$ to $130^{\circ} \mathrm{C}$ the switching field of the memory layer is negative, i.e. the switching occurs before 


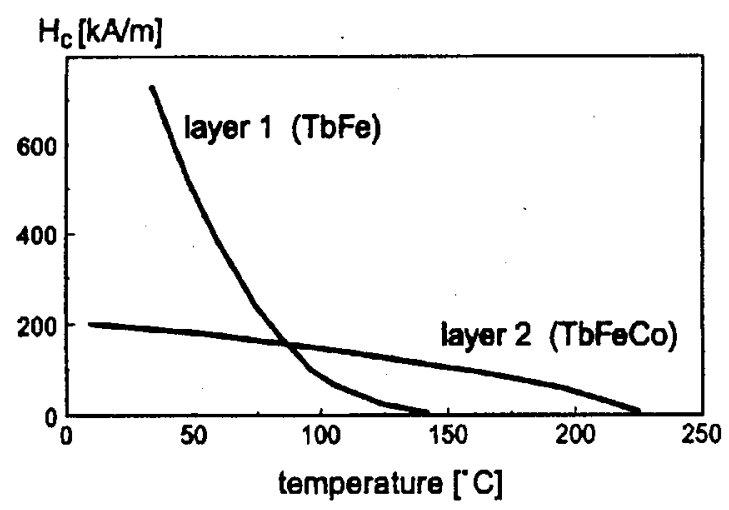

Fig. 5. Coercivity $H_{\mathrm{c}}$ as a function of temperature for a TM-dominated layer (1) and a RE-dominated layer (2).

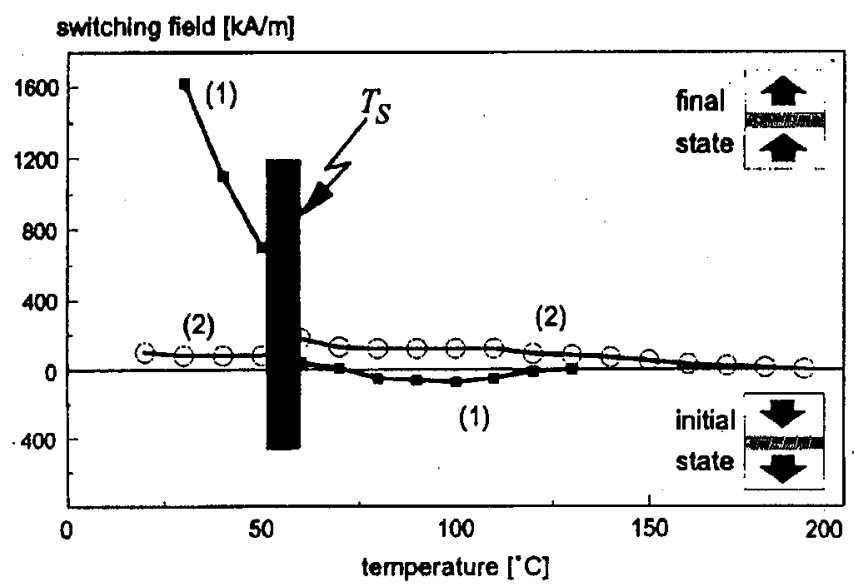

Fig. 6. Switching fields of an ECDL with antiparallel coupling as a function of temperature; layer (1) is TM-dominated and layer (2) RE-dominated. The data were measured by changing the external field from negative saturation (initial state) to positive saturation (final state) at constant temperature.

the field is reversed (as already shown in Fig. 3). It is caused by the wall energy, which allows switching against the external field [9-11].

The switching behaviour of Fig. 6 can be understood, when the switching fields are discussed in terms of Eq. (1). Figure 7 shows a graphical representation of Eq. (1) $[10,11]$. Without coupling energy the switching curves would be given by the coercivities $H_{\mathrm{c} 1}$ and $H_{\mathrm{c} 2}$. Due to the coupling energy each of these curves splits into two branches $H_{\mathrm{s}}^{ \pm}$, which correspond to the additional effect of creation or annihilation of an interface wall. Above the Curie temperature $T_{\mathrm{c} 1}$ of the memory layer no coupling and, hence, no splitting of the curves exists.

Only a few of the various branches are observed during a complete magnetic 


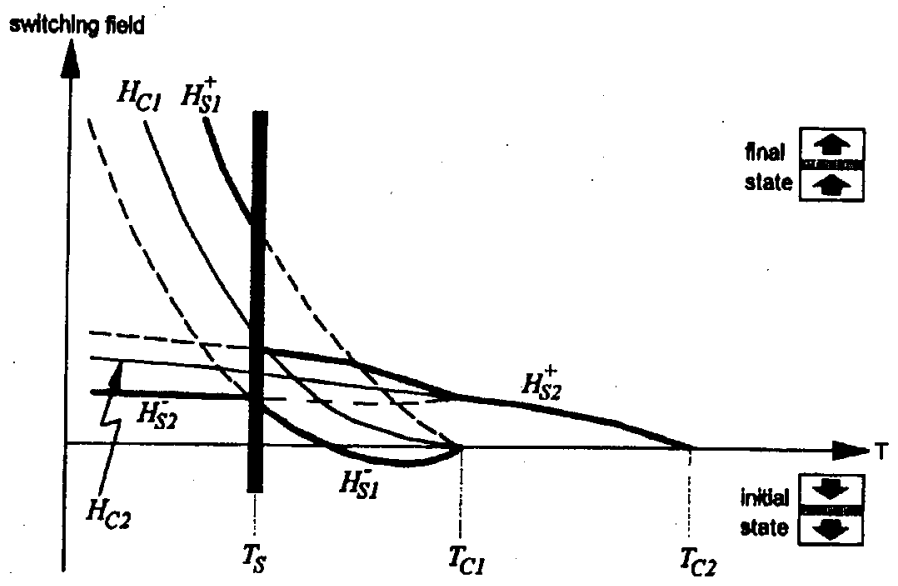

Fig. 7. Switching field diagram of an ECDL with antiparallel coupling; the diagram is a graphical interpretation of Eq. (1) and explains the experimental data of Fig. 6.

reversal $[10,11]$. Let us consider a situation below the critical temperature $T<T_{\mathbf{s}}$. In a negative saturation field an interface wall exists, which is annihilated during the first switching process. It occurs at the lowest switching field $H_{s}^{-}$available which is $H_{\mathrm{s} 2}^{-}$. Then, the magnetizations of layers (2) and (1) are up and down, respectively, and no interface wall exists. Next switching in a positive field takes place with layer (1) and requires formation of the interface wall again. Switching, therefore, occurs at the switching field $H_{\mathbf{s} 1}^{+}$. For temperatures above the critical temperature $T_{\mathbf{s}}$ a similar discussion explains the fact that now firstly layer (1) switches by annihilation and then layer (2) by creation of an interface wall. Here, the switching fields are $H_{\mathrm{s} 1}^{-}$and $H_{\mathrm{s} 2}^{+}$, respectively. In a small temperature range near $T_{\mathbf{s}}$ a more complicated switching behaviour takes place [8-11].

\section{Application of the switching field diagram}

The switching diagram can be used to determine quantitatively the temperatures $T_{\mathrm{L}}$ and $T_{\mathrm{H}}$ and the fields $H_{\mathrm{b}}$ and $H_{\mathrm{ini}}$ required for a reliable DOW process. The diagram can be interpreted as a phase diagram, where the switching field lines separate regions of stable magnetization up $(\uparrow)$ and down $(\downarrow)[10,11]$. An original magnetization down $(\downarrow)$ switches up $(\uparrow)$, when an external field $H$ is applied which is above the relevant switching lines $\mathrm{H}_{\mathrm{s}}^{+}$or $\mathrm{H}_{\mathrm{s}}^{-}$. When the original magnetization is up ( $\uparrow$ ), the branches of the switching diagram (Fig. 7) have to be inverted with respect to sign. Then, switching occurs if the external field is below the appropriate switching field line.

As an example, the high temperature procedures of a DOW cycle will be discussed for ECDLs with antiparallel coupling (Fig. 8). Near the Curie temperature $T_{\mathrm{c} 2}$ a domain is formed in layer (2) by the bias field $H_{\mathrm{b}}$. During cooling eventually the Curie temperature $T_{\mathrm{c} 1}$ of the memory layer (1) is reached. Then a domain has to be formed also in the memory layer by the exchange coupling in a direction opposite to the (negative) direction of $H_{\mathrm{b}}$. This can only occur when a 


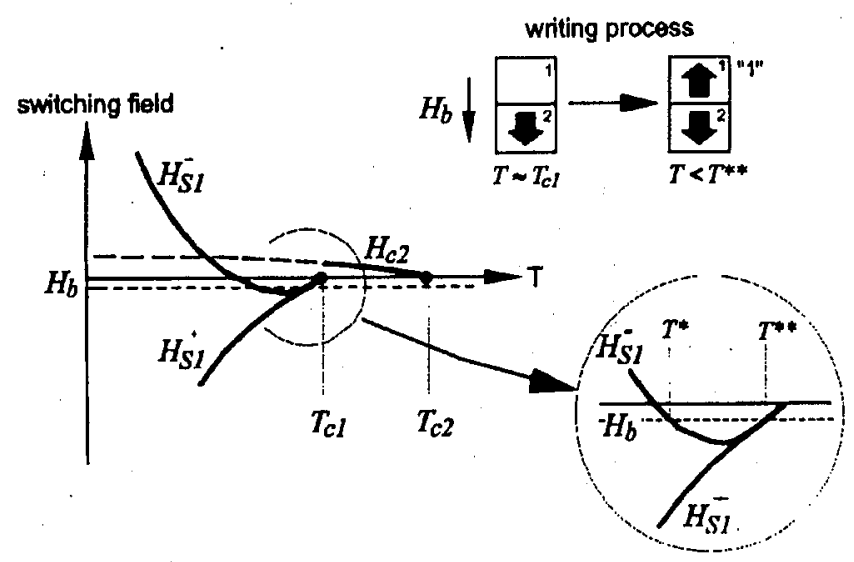

Fig. 8. Application of the switching field diagram to the high temperature writing cycle; a domain is formed in layer (2) at the high temperature $T_{\mathrm{H}} \approx T_{\mathrm{c} 2}$; after cooling to $T_{\mathrm{c} 1}$ a domain representing information " 1 " has to be formed by exchange coupling, which must be stable against the negative bias field $H_{\mathrm{b}}$ for $T<T^{* *}$.

temperature range exists, where $H_{\mathrm{b}}$ is above the curve $H_{\mathrm{s} 1}^{-}$, i.e. in the temperature range between $T^{* *}$ and $T^{*}[10,11]$. Therefore, the negative part of the switching field $H_{\mathrm{s} 1}^{-}$already shown in Figs. 3, 6 and 7 is essential for the high temperature writing process. Even the dynamical behaviour of the process can be analyzed if the fact is taken into account that the bias field $H_{\mathrm{b}}$ decreases with time because of the rotation of the disk.

Similar discussion can be made for all steps of the DOW cycle. The parameters to fulfil the criteria must be adjusted according to Eq. (1). The magnetic moment $\left(M_{\mathrm{s}} t\right)$ per unit area can be changed by the composition, which influences $M_{\mathrm{s}}$, and by the thickness $t$. It is difficult, however, to adjust other magnetic parameters independently because of the correlations of the sputtering process. Increasing the thickness, for instance, requires higher sputtering power or larger sputtering time which influences the temperature and, as a consequence, the wall energy [12].

The various steps leading to a DOW process can be simulated by experiments in a macroscopic scale, where the double layer is exposed to appropriate fields and temperatures and the magnetization of layers (1) and (2) are determined by Kerr measurements $[13,14]$. Figure 9 shows the results of a low temperature cycle. At first, the hysteresis loops of the memory layer (1) and of the reference layer (2) were determined at room temperature. Let us start with configuration $A$ representing the infomation " 1 ". An initialization field of about $200 \mathrm{kA} / \mathrm{m}$ saturates the reference layer, but does not significantly change the memory layer (configuration $B$ ). A small negative bias field $H_{\mathrm{b}}$ applied at room temperature $(C)$ does not change the magnetization configuration. If the temperature is increased up to $T_{\mathrm{L}}=140^{\circ} \mathrm{C}$, the magnetization of the reference layer $(2)$ is slightly reduced $(D)$, but the memory layer (1) is heated to the Curie temperature, where the magnetization is zero. Cooling in a negative field induces a reversed magnetization 

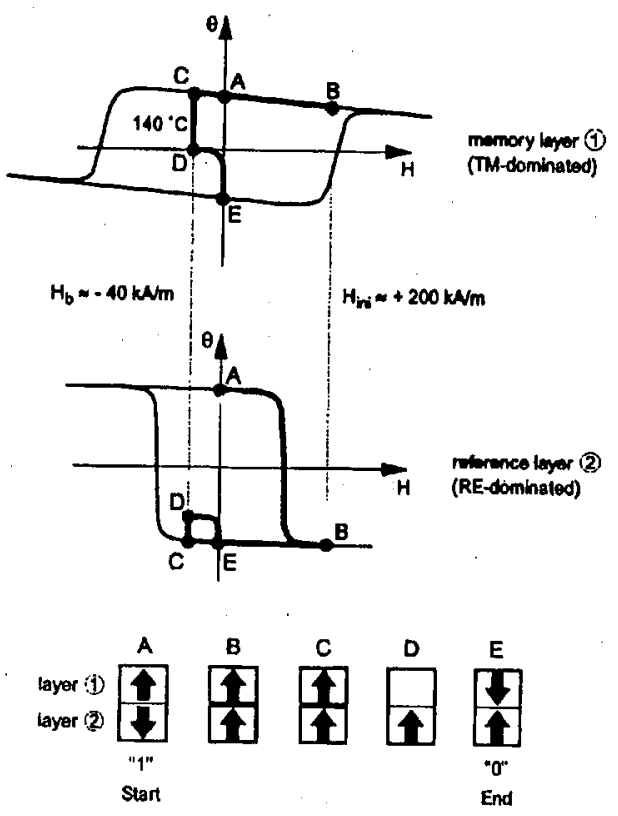

Fig. 9. Macroscopic simulation of a low temperature writing cycle at $T_{\mathrm{L}}=140^{\circ} \mathrm{C}$; the magnetic configurations of the memory layer (1) and the reference layer (2) were determined by Kerr measurements at various steps $A-E$.

in layer (1) because of exchange coupling. The final situation is $(E)$, where the configuration corresponds to the information " 0 " (cf. Fig. 4).

Experiments like that allow to follow macroscopically the various steps of the switching process and provide quantitative numbers for the necessary fields and temperatures. In the previous sections only double layers with antiparallel coupling were discussed. Similarly, also parallel coupling in ECDLs can be applied. In this case, both layers are dominated either by the RE components or by the TM components. Various systems were proposed which contain three or more layers. In all cases the parallel or antiparallel coupling between ECDLs is the essential mechanism which can be studied quantitatively by the switching field formalism.

\section{Magnetically induced superresolution (MSR)}

Exchange coupled double layers can also be applied to achieve a resolution beyond the optical diffraction limit. According to optical diffraction theory the diameter of the focused laser beam is given by $d=1.22(\lambda / A)$, where $A$ is the numerical aperture of the optical system. In magneto-optical recording the size of the written domain can be smaller than $d$, because it is determined by the temperature profile induced by the laser pulse. The reading process, however, is still limited by diffraction theory, because the maximum of one diffraction spot should fall at least into the minimum of the next spot.

Domains with a smaller distance can be read when part of the information is optically masked (magnetically induced superresolution) (Fig. 10). Then the ef- 

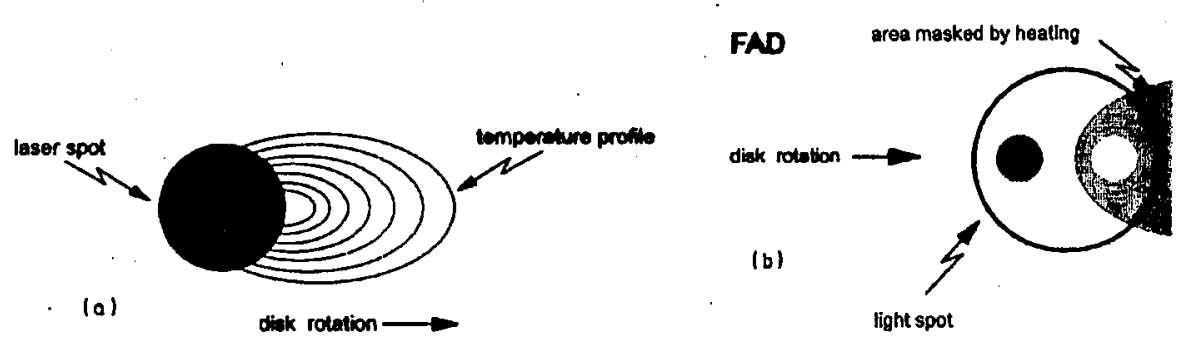

Fig. 10. Principle of magnetic superresolution: (a) temperature profile caused by the laser spot on a moving disk; (b) front aperture detection by masking a mark at the front of the laser spot.

fective aperture is smaller than that given by optical theory and the information can be extracted from the intensity modulation induced by the rotating disk [15]. Masking can be obtained, in principle, by the temperature profile of the reading laser. Due to disk rotation, the effective temperature profile in the magneto-optical layer is elliptical with a maximum near the front edge of the beam spot (Fig. 10a). If the maximum temperature is sufficient for erasing, only the mark in the low temperature crescent remains (Fig. 10b) and contributes to the signal (front aperture detection, FAD).

Exchange coupled layer systems can be prepared which allow this type of reading in a readout layer without loosing the information stored in an additional recording layer. In this case, the information is copied from the recording layer back to the readout layer by exchange coupling during the cooling after the laser pulse. Alternatively, masking can be obtained also in other parts of the laser spot by appropriate techniques $[3,5]$. All this ideas make use of the fact that energy can be stored or released in the interface between two ECDLs by appropriate procedures. The switching field formalism, which has been described for the DOW, can be applied in a similar way also to the MSR procedures, because they are based on the same principles for switching $[10,11]$. This is important, because the MSR procedure is very sensitive to the external parameters. The temperature gradient in the irradiated area, for instance, plays an important role and has to be adjusted even more precisely than in DOW. The switching field formalism can help to find the appropriate temperature and field ranges from macroscopic experiments.

\section{Outlook}

The present a vailable $5.25^{\prime \prime}$ magneto-optical disks have a capacity of $650 \mathrm{MB}$ per side (capacity 2X), which corresponds to the standard of the CD-ROM. As a disk can be used from both sides, the total capacity is $1.3 \mathrm{~GB}$. The data are recorded in marks of $0.65 \mu \mathrm{m}$ diameter in a distance of $1.29 \mu \mathrm{m}$ along the track and at a track pitch of $1.4 \mu \mathrm{m}$ [16]. The detection is done by a $780 \mathrm{~nm}$ laser by using the pulse position modulation (PPM) code to obtain an area density of $410 \mathrm{Mbit}^{\mathrm{inch}}{ }^{2}$ on average.

Lasers with shorter wavelengths available in future may lead to a further increase in capacity. Compared with the present standard the blue laser $(428 \mathrm{~nm})$ 
will supply, for instance, a factor 3.8 by a linear reduction of the dimensions [16]. In this case, however, new materials such as CoPt multilayers [17] are required, because the RE/TM materials give only a low signal-to-noise ratio at short wavelengths.

The exchange coupled layer systems, on the other hand, provide a great variety of solutions to obtain direct overwrite and magnetic superresolution without substantial changes of materials and techniques. In prototypes, for instance, a recording density of over 2 GBit/inch ${ }^{2}$ has been achieved by means of MSR in a combination with land groove recording using a conventional red laser $(680 \mathrm{~nm})$ [18]. Although none of these techniques is applied in a present technical product, some of the various approaches will certainly be incorporated into further developments.

\section{Acknowledgment}

The authors are indebted to T.M. Atmono, M. Kratzer and E. Bruehl for valuable contributions and technical assistance.

\section{References}

[1] D.S. Bloomberg, G.A.N. Conell, recording, in: Magnetic Recording Technology, Eds. C.D. Mee, E.D. Daniels, Vol. 3, San Diego 1988, Ch. 6.

[2] M. Mansuripur, The Physical Principles of Magneto-Optical Recording, Cambridge Univ. Press, Cambridge 1995.

[3] K. Roell, in: Magnetic Thin Films and Industrial Application, Ed. U. Hartmann, Springer Series in Material Sciences, Springer, Berlin 1996.

[4] P. Hansen, J. Magn. Magn. Mater 83, 6 (1990).

[5] P. Hansen, in: IIandbook of Magnetic Materials, Vol. 6, Ed. K.H.J. Buschow, Elsevier, Amsterdam 1991, Ch. 4, p. 289.

[6] J. Saito, M. Sato, H. Matsumoto, H. Akasaka, Jpn. J. Appl. Phys. 26, suppl. 26 (1987).

[7] T. Kobayashi, H. Tsuji, S. Tsunashima, S. Uchiyama, Jpn. J. Appl. Phys. 20, 2089 (1981).

[8] D. Mergel, J. Appl. Phys. 74, 4072 (1993).

[9] F. Stobiecki, T.M. Atmono, S. Becker, H. Rohrmann, K. Roell, J. Magn. Magn. Mater. 148, 497 (1995).

[10] S. Becker, Ph.D. Thesis, Univ. Kassel, 1995.

[11] S. Becker, H. Rohrmann, K. Roell, submitted to J. Magn. Magn. Mater.

[12] D. Raasch, J. Magn. Magn. Mater. 101, 202 (1991).

[13] T.M. Atmono, Ph.D. Thesis, Univ. Kassel, 1992.

[14] S. Becker, P.W.M. Filbrandt, C.D. Wright, H. Rohrmann, W.W. Clegg, K. Roell, J. Magn. Magn. Mater. 148, 241 (1995).

[15] M. Kaneko, K. Aratani, M. Ohta, Jpn. J. Appl. Phys. 31, 568 (1992).

[16] T. Suzuki, Camst-Read/Write 95-22, 15 (1995).

[17] F.J.A.M. Greidanus, W.B. Zeper, F.J.A. den Broeder, W.F. Godlieb, Appl. Phys. Lett. 54, 2481 (1989).

[18] A. Takahashi, J. Magn. Soc. Jpn. 19, S1, 273 (1995). 